\title{
Zukunft der Arbeit im Kontext von Autonomik und Industrie 4.0
}

\author{
Alfons Botthof
}

Mit dem Zukunftsprojekt „Industrie 4.0“, das ein zentrales Element der Hightech-/ Innovations-Strategie der Bundesregierung darstellt, soll die Informatisierung der klassischen Industrien, wie z. B. der Produktionstechnik, vorangetrieben werden (Abb. 1). „Auf dem Weg zum Internet der Dinge soll durch die Verschmelzung der virtuellen mit der physikalischen Welt zu Cyber-Physical Systems und dem dadurch möglichen Zusammenwachsen der technischen Prozesse mit den Geschäftsprozessen der Produktionsstandort Deutschland in ein neues Zeitalter geführt werden.“1

\section{Handlungsfelder Industrie 4.0}

- Sicherheit als erfolgskritischer Faktor

- Rechtliche Rahmenbedingungen

- Arbeitsorganisation und Arbeitsgestaltung im digitalen Industriezeitalter

- Normung, Standardisierung und offene Standards für eine Referenzarchitektur

- Beherrschung komplexer Systeme

- Flächendeckende Breitbandinfrastruktur für die Industrie

- Aus- und Weiterbildung

- Ressourceneffizienz

- Neue Geschäftsmodelle

Quelle: Arbeitskreis Industrie 4.0 (Forschungsunion, acatech):

Umsetzungsempfehlungen für das Zukunftsprojekt Industrie 4.0, April 2013

\footnotetext{
${ }^{1}$ Promotorengruppe Kommunikation der Forschungsunion Wirtschaft - Wissenschaft (Hg.): Im Fokus: Das Zukunftsprojekt Industrie 4.0 - Handlungsempfehlungen zur Umsetzung, März 2012, S. 8.
}

Der Originaltext dieses Beitrags wurde überarbeitet. Das vollständige Korrekturverzeichnis finden Sie am Ende des Buchs und online unter http://dx.doi.org/10.1007/978-3-662-45915-7_16.

A. Botthof $(\bowtie)$

Institut für Innovation und Technik (iit), Berlin, Germany

e-mail: botthof@iit-berlin.de

(C) The Author(s) 2015

A. Botthof, E.A. Hartmann (Hrsg.), Zukunft der Arbeit in Industrie 4.0,

DOI 10.1007/978-3-662-45915-7_1 


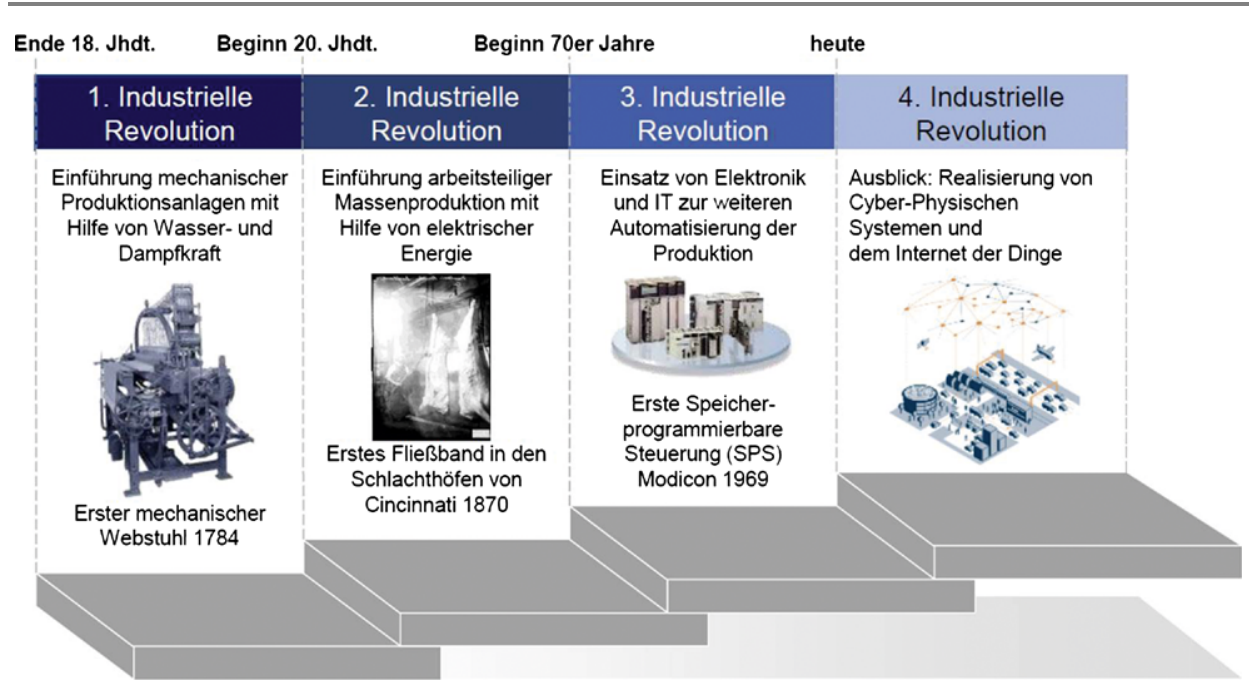

Abb. 1 Industrie 4.0; Quelle: Picot/Münchner Kreis, in Anlehnung an Forschungsunion (2012)

Verfolgte man den Prozess der Diskussion rund um das Zukunftsprojekt Industrie 4.0, zunächst im Kreis der Promotorengruppe Kommunikation innerhalb der Forschungsunion und dann in Vertiefung im gleichnamigen Arbeitskreis unter dem Vorsitz von Henning Kagermann (Deutsche Akademie der Technikwissenschaften - acatech) und Siegfried Dais (Robert Bosch Industrietreuhand KG), so konnte man feststellen, dass sehr intensiv auch über die Wirkungen von Industrie 4.0 auf die Qualität der Arbeit, die Qualifikationserfordernisse, neue Formen der Arbeitsorganisation und Veränderungen im Zusammenspiel zwischen Mensch und Technik nachgedacht wurde. Zunächst unter der nicht ganz glücklich gewählten Überschrift „Faktor Mensch““, dann „Mensch und Arbeit“ befasste man sich mit dem absehbaren Paradigmenwechsel in der Mensch-Technik- und Mensch-Umgebungs-Interaktion und den damit verbundenen neuartigen Formen der kollaborativen Fabrikarbeit. In der Überzeugung, dass auch die Smart Factory im Rahmen von Industrie 4.0 keineswegs menschenleer sein wird, wurden zudem die Anforderungen an die Fähigkeiten und das Wissen von Beschäftigten in einem sich verändernden Arbeitsumfeld, bestimmt von komplexen Prozessen, technologisch anspruchsvollen Anlagen und Werkzeugen, ausführlich thematisiert. Neben kurz- und mittelfristigen Handlungsfeldern (bspw. Assistenzsysteme als „Fähigkeitsverstärker“ physischer und kognitiver Leistungen, kollaborative industrielle Serviceroboter, Apps für eine software-basierte Konfiguration von Anlagen oder auch AR-Technologien zur schnellen Einweisung in Fertigungsprozesse oder zur Lernunterstützung) wurde eine Qualifizierungsinitiative vorgeschlagen, die sowohl die gewerbliche als auch hochschulische Aus- und Weiterbildung adressiert.

\footnotetext{
${ }^{2}$ Ebenda S. $33 \mathrm{ff}$.
} 
Dieser Themenkomplex ruft traditionell auch die Sozialpartner auf den Plan, die jeder für sich erkannt haben, dass Industrie 4.0 oder - etwas allgemeiner formuliert - der Trend zur zunehmenden Informatisierung der Arbeitswelt potenziell starke Auswirkungen auf die Beschäftigten und deren Situation in den Betrieben generell und spezifisch auf Formen der Arbeitsorganisation haben wird. Dies betrifft insbesondere die Qualität der Arbeit einschließlich Faktoren wie Arbeitszufriedenheit und Gesundheit - sowie das allgemeine Qualifikationsniveau wie auch die spezifisch notwendigen Qualifikationen und Kompetenzentwicklungsprozesse.

Bereits vom Juli 2009 liegt ein Arbeitspapier der Gewerkschaften zum Internet der Dinge und der Informatisierung der Arbeitswelt und des Alltags vor. ${ }^{3}$ Sehr ausgewogen wurden hier im Anwendungsfeld „Produktion - Fertigungsplanung“ negative wie positive Wirkungen diskutiert. So werden hier beispielsweise durch die Automatisierung und Dezentralisierung von Steuerungsprozessen („Halbzeuge“ und Komponenten tragen die Information über die nächsten Prozessschritte mit sich und kommunizieren autark mit Bearbeitungswerkzeugen und Fertigungsstraßen) einerseits Verluste an Kontroll- und Steuerungsmöglichkeiten befürchtet andererseits aber auch die Chancen einer besseren Anpassung der Gesamtprozesse an individuelle Anforderungen. „Welche konkreten Auswirkungen für die Beschäftigten auftreten hängt sehr stark von den jeweils konkret realisierten Organisations- und Technikkonzepten der Betriebe ab. Sowohl hinsichtlich der Technik wie hinsichtlich der Organisation gibt es Alternativen, zwischen denen gewählt werden kann. Notwendig sind konkrete Technik- und Organisationsentwicklungsprojekte, die dazu dienen, die Machbarkeit und Wirtschaftlichkeit von menschenzentrierten Betriebsund Technikmodellen zu demonstrieren. “4

Diese Reflexionen innerhalb der Gewerkschaften wurden u. a. ausgelöst durch Diskussionen auf europäischer Ebene ${ }^{5}$ zu neuen Modellen der Organisation von Unternehmen und unternehmensübergreifenden Netzen bis hin zur „fabriklosen Fertigung“, der individualisierten Massenfertigung sowie den sich selbst konfigurierenden oder gar selbst organisierenden Produktionssystemen, wie diese auch als Szenario in Industrie 4.0 beschrieben werden.

In den Handlungsempfehlungen des Abschlussberichts des Arbeitskreises Industrie 4.0, an denen Gewerkschaftsvertreter mitwirkten, finden sich die zentralen Zielsetzungen, die im Rahmen einer Arbeitsgruppe der Plattform Industrie 4.0 verfolgt werden sollen:

\footnotetext{
${ }^{3}$ Botthof, A., Bovenschulte M. (Hg.): Das „Internet der Dinge“ - Die Informatisierung der Arbeitswelt und des Alltags; Arbeitspapier 176, im Auftrag der Hans Böckler Stiftung, Juli 2009.

${ }^{4}$ Ebenda S. 32.

${ }^{5}$ So z. B. die Roadmap resp. Strategic Research Agenda der europäischen Technologieplattform MANUFUTURE (siehe www.manufuture.org).
} 
So wird man sich inhaltlich befassen mit

- den Auswirkungen für Arbeit und Beschäftigung (Chancen und Risiken) sowie Handlungsbedarfe im Hinblick auf eine beschäftigtenorientierte Arbeits- und Qualifizierungspolitik.

- Orientierungs- und Handlungshilfen für die Weiterentwicklung und Implementierung des sozio-technischen Gestaltungsansatzes sowie entsprechender Referenzprojekte.

- innovativen Ansätzen partizipativer Arbeitsgestaltung und lebensbegleitender Qualifizierungsmaßnahmen, die über Altersgruppen, Geschlecht und Qualifikationsniveaus hinweg die ganze Breite der Belegschaften berücksichtigen.

Darüber hinaus wird ein regelmäßiger Dialog zwischen den Sozialpartnern empfohlen, in dem wichtige Fortschritte, Problemfelder und Lösungsmöglichkeiten bei der Umsetzung von Industrie 4.0 transparent gemacht und beraten werden. ${ }^{6}$

In diesem thematischen Umfeld hat die vom Bundesministerium für Wirtschaft und Energie beauftragte Begleitforschung zum Technologieprogramm Autonomik ${ }^{7}$ in der Befassung mit den geförderten FuE-Vorhaben das Handlungsfeld „Mensch-TechnikInteraktion“ als ein wichtiges Querschnittsthema identifiziert. Folgende Fragenkomplexe wurden hier $\mathrm{u}$. a. behandelt: ${ }^{8}$

- Wie wird ein autonomes System/ein autonomer Prozess in die Arbeitsorganisation integriert?

- Welche Auswirkungen sind hinsichtlich Arbeitsinhalte, Aufgaben, Verantwortungsbereiche der Nutzer zu erwarten?

- Wie könnte im Hinblick auf das körperliche und geistige Leistungsvermögen der Nutzer und der Entwicklung dieses Leistungsvermögens eine günstige Arbeitsorganisation aussehen?

- Welche Gestaltungsanforderungen und -optionen ergeben sich für autonome Systeme?

- Welche Gestaltungskriterien können aus der Sicht der Mensch-Technik-Interaktion für autonome Systeme formuliert werden?

- Welche Erkenntnisse und methodische Hilfsmittel gibt es, auf denen aufbauend Fragen der Mensch-Technik-Interaktion im Entwicklungs- und Gestaltungsprozess effektiv und effizient adressiert werden können?

\footnotetext{
${ }^{6}$ Kagermann, H., Wahlster, W., Helbig, J. (Hg.): Umsetzungsempfehlungen für das Zukunftsprojekt Industrie 4.0, Abschlussbericht des Arbeitskreises Industrie 4.0; April 2013; S. 58.

${ }^{7}$ Die Begleitforschung verantwortete das Institut für Innovation + Technik (iit) in Berlin; Laufzeit des Technologieprogramms „AUTONOMIK - autonome und simulationsbasierte Systeme für den Mittelstand": 2009 bis 2013.

${ }^{8}$ Die gewonnenen Erkenntnisse und daraus abgeleitete Empfehlungen sind zusammengefasst in: Bundesministerium für Wirtschaft und Technologie (Hg.): Mensch-Technik-Interaktion - Leitfaden für Hersteller und Anwender; Berlin, Jan. 2013.
} 
Diese Fragestellungen werden im laufenden Technologieprogramm des BMWi, ,Autonomik für Industrie 4.0“, erweitert und auf industrielle Prozesse hin konkretisiert. Zusammen mit dem Vorläuferprogramm „Autonomik - autonome und simulationsbasierte Systeme für den Mittelstand“ versteht sich „Autonomik für Industrie 4.0“ als Wegbereiter der vierten industriellen Revolution und stellt den zentralen Beitrag des Wirtschaftsministeriums zum Zukunftsprojekt Industrie 4.0 der Bundesregierung dar.

Konsequenter noch als durch seinen Vorgänger sollen mit dem Technologieprogramm „AUTONOMIK für Industrie 4.0“9 modernste I\&K-Technologien mit der industriellen Produktion unter Nutzung von Innovationspotenzialen verzahnt und die Entwicklung innovativer Produkte und Prozesse beschleunigt werden. Übergeordnetes, wirtschaftspolitisches Ziel ist es, Deutschlands Spitzenstellung als hochwertiger Produktionsstandort und als Anbieter für modernste Produktionstechnologien zu stärken.

Hierzu gehen exzellente, im Technologieprogramm sorgsam ausgewählte Verbundvorhaben an den Start, denen allesamt ein hohes Innovationspotenzial zugeschrieben wird.

Die geförderten Vorhaben in den Feldern Produktion, Logistik und Robotik befassen sich u. a. mit

- mobilen Assistenzsystemen und Internetdiensten,

- der Plug\&Play-Vernetzung von Maschinen und Anlagen,

- autonom handelnden, fahrerlosen Transportfahrzeugen,

- bionisch gesteuerten Fertigungssystemen für die Herstellung kundenindividueller Produkte,

- mit Schutz- und Sicherheitskonzepten für die Zusammenarbeit von Mensch und Roboter in gemeinsamen Arbeitsbereichen,

- der Inventur von Lagerbeständen mit autonomen Flugrobotern,

- der Planungs- und Entscheidungsunterstützung bei der Auswahl industrieller Serviceroboter,

- autonomer Echtzeitassistenz für Fertigungsmitarbeiter,

- 3D-gestützter Engineering-Plattform für die intuitive Entwicklung und effiziente Inbetriebnahme von Produktionsanlagen,

- der Plug\&Play-Integration von Robotern in der Industrieautomatisierung,

- der dezentralen Produktionssteuerung für die Automobilindustrie und

- der automatischen Einzelstückfertigung von Sportschuhen und Textilien.

Nahezu alle Projektkontexte weisen einen hohen Bezug zum Thema „Veränderungen in der Arbeitswelt" auf und versprechen hohe Aufmerksamkeit zu erzielen sowie Breitenwirkung in der ,community of practice“ zu Industrie $4.0 \mathrm{zu}$ entfalten.

Es wird sich lohnen, die Vorhaben mit den in dieser Veröffentlichung zusammengetragen Erfahrungen der Autonomik-Vergangenheit, den Erkenntnissen der Arbeitswissenschaften zu konfrontieren und als konkrete Anwendungsfälle in die Diskussionen bspw.

\footnotetext{
${ }^{9}$ Siehe www.autonomik40.de.
} 
der Plattform Industrie 4.0 einzubringen. Dabei steht zu erwarten, dass Thesen und Befunde validiert, Erkenntnisse weiter präzisiert und gute Praktiken entwickelt und kommuniziert werden können. Einerseits wird zu prüfen sein, welche vorhandenen Wissensbestände zur Arbeitsorganisation und zur Arbeitsgestaltung prinzipiell auch auf den Einsatz autonomer Systeme in der Industrie 4.0 anzuwenden sind. Andererseits gilt es die Entstehung ganz neuartiger Herausforderungen durch autonome Cyber-Physikalische Systeme genauer zu analysieren.

Neben dem Thema „Zukunft der Arbeit“ richten sich begleitende Forschungs- und Unterstützungsmaßnahmen im Technologieprogramm unter Einbindung einschlägiger Experten und Interessenten aus Industrie und Wissenschaft auf weitere Themen, die Einfluss nehmen auf Innovationsprozesse und -geschwindigkeiten im Innovationsfeld „Industrie 4.0“. Als innovationsunterstützende und -beschleunigende Maßnahmen stehen diese für ein holistisches Verständnis der Innovationsförderung, die nicht alleine auf technologische FuE-Projekte und prototypische Lösungen zielt. Einige dieser erfolgskritischen Innovationshemmnisse weisen in Ausprägungen auch Querbezüge zum Thema „Arbeit“" auf. Folgende Handlungsfelder seien beispielhaft genannt, die in den Autonomik-Programmen durch die Begleitforschung prioritär analysiert und mit Aktivitäten der zuständigen resp. betroffenen Stakeholder untersetzt werden:

\section{- Rechtliche Herausforderungen}

Anforderungen an den Arbeits- und Datenschutz, der rechtskonforme und sichere Einsatz autonomer Systeme, straf-/zivilrechtliche Haftung, Betriebszulassung/Zertifizierung, Risk Management - Versicherungsfähigkeit, Fragen der sensiblen Weiterentwicklung des Rechtssystems.

\section{- Normung und Standardisierung}

Normen und Standards als Grundlage für Industrie 4.0, Entwicklungsbegleitende Normung, Referenzarchitekturen, Konkretisierung und Weiterentwicklung einer nationalen Normungsroadmap.

\section{- IT-Sicherheit für Industrie 4.0}

Schutz der IT-basierten Infrastruktur vernetzter Maschinen und Anlagen zur Automatisierung, Überwachung und Steuerung von industriellen Prozessen vor Spionage und Sabotage. Security-by-design, neue Sicherheitsarchitekturen, Einhaltung von Verhaltensmaßregeln, Gesetzen und Richtlinien (Compliance) mit arbeitsorganisatorischen Implikationen.

Open Access This chapter is distributed under the terms of the Creative Commons Attribution Noncommercial License, which permits any noncommercial use, distribution, and reproduction in any medium, provided the original author(s) and source are credited. 